\section{LA-UR- $97-265 \%$ \\ Aooroved for public release} atstribution is unlimited.
Title:

Post-Scission Fission Theory: Neutron Emission in Fission

Submitted to:

David G. Madland

Fen

mov 31937

01

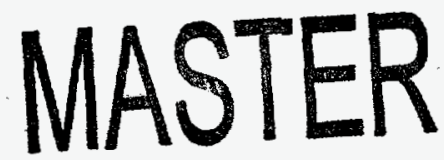

Proceedings of the Workshop on Nuclear Reaction Data and Nuclear Reactors - Physics, Design and Safety

\title{
NATIONAL LABORATORY
}

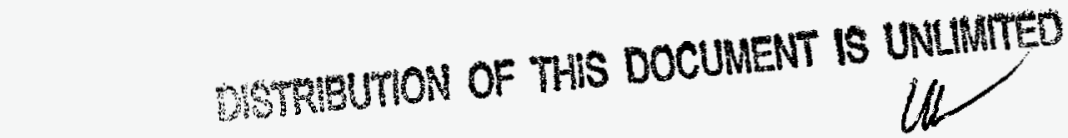

Los Alamos National Laboratory, an affirmative actionlequal opportunity employer, is operated by the University of Califomia for the U.S. Department of Energy under contract W-7405-ENG-36. By acceptance of this article, the publisher recognizes that the U.S. Goverment retains a nonexclusive, royalty-free license to publish or reproduce the published form of this contribution, or to allow others to do so, for U.S. Government purposes. Los Alamos National Laboratory requests that the publisher identify this article as work performed under the auspices of the U.S. Department of Energy. The Los Alamos National Laboratory strongly supports academic freedom and a researcher's right to publish; as an institution, however, the Laboratory does not endorse the viewpoint of a publication or guarantee its technical correctness. 


\section{DISCLAMMER}

Portions of this docament may be illegible in electronic image products. Images are produced from the best available original document. 


\section{DISCLAIMER}

This report was prepared as an account of work sponsored by an agency of the United States Government. Neither the United States Government nor any agency thereof, nor any of their employees, make any warranty, express or implied, or assumes any legal liability or responsibility for the accuracy, completeness, or usefulness of any information, apparatus, product, or process disclosed, or represents that its use would not infringe privately owned rights. Reference herein to any specific commercial product, process, or service by trade name, trademark, manufacturer, or otherwise does not necessarily constitute or imply its endorsement, recommendation, or favoring by the United States Government or any agency thereof. The views and opinions of authors expressed herein do not necessarily state or reflect those of the United States Government or any agency thereof. 


\title{
POST-SCISSION FISSION THEORY: NEUTRON EMISSION IN FISSION
}

\author{
David G. Madland \\ Theoretical Division, Los Alamos National Laboratory \\ Los Alamos, New Mexico 87545 USA
}

\begin{abstract}
A survey of theoretical representations of two of the observables in neutron emission in fission is given, namely, the prompt fission neutron spectrum $N(E)$ and the average prompt neutron multiplicity $\bar{\nu}_{p}$. Early representations of the two observables are presented and their deficiencies are discussed. This is followed by summaries and examples of recent theoretical models for the calculation of these quantities. Emphasis is placed upon the predictability and accuracy of the recent models. In particular, the dependencies of $N(E)$ and $\bar{\nu}_{p}$ upon the fissioning nucleus and its excitation energy are treated. Recent work in the calculation of the prompt fission neutron spectrum matrix $N\left(E, E_{n}\right)$, where $E_{n}$ is the energy of the neutron inducing fission, is then discussed. Concluding remarks address the current status of our ability to calculate these observables with confidence, the direction of future theoretical efforts, and limitations to current (and future) approaches.
\end{abstract}

\section{Observables in Neutron Emission in Fission}

The observables in neutron emission in fission include the following:

(a) Time dependence of neutron emission. The classical single-particle orbit time in a medium-mass nucleus is of the order of $10^{-21}$ seconds. The time neccessary for an actinide fission fragment to reach $90 \%$ of its final kinetic energy due to Coulomb repulsion is of the order of $10^{-20}$ seconds, about 10 orbit times. Fission-fragment compound nucleus lifetimes range from about $10^{-18}$ to $10^{-13}$ seconds, or about $10^{3}$ to $10^{8}$ orbit times, respectively. Neutrons and gamma rays emitted up to this point are referred to as "prompt" neutrons and "prompt" gamma rays from fission fragments. They are emitted under the actions of the strong and electromagnetic forces, respectively. Neutrons emitted following $\beta$ decay from the action of the weak force which is much slower, are correspondingly held back and are referred to as "delayed" neutrons emitted from fission products, where a fission "product" is a fission fragment with completed prompt neutron emission.

(b) The energy spectrum of the emitted prompt neutrons. This is the prompt fission neutron energy spectrum $N(E)$ where $E$ is the kinetic energy of the emitted neutron in the laboratory system and the normalization of the spectrum is usually defined by the equation

$$
\int_{0}^{\infty} N(E) d E=1
$$


(c) The average number of prompt neutrons emitted per fission. This is called the average prompt fission neutron multiplicity $\bar{\nu}_{p}$.

(d) The distribution of the number of prompt neutrons emitted per fission whose average is $\bar{\nu}_{p}$. This is called the prompt fission neutron multiplicity distribution $P(\nu)$.

(e) Correlations in neutron emission yields $\nu$ from complementary fission fragments.

(f) Energy spectra and multiplicities of pre-fission neutrons. These are neutrons emitted prior to the fission event in multiple-chance fission. They are generally produced via equilibrium, preequilibrium, or direct (knockout) reaction mechanisms.

(g) Energy spectra and multiplicities of scission neutrons. These are neutrons in coincidence with the fission event that are emitted isotropically in the laboratory frame and are therefore associated with the fissioning system just before the nascent fission fragments separate at the scission point.

\section{Early Representations of $N(E)$ and $\bar{\nu}_{p}$}

Two early representations of the prompt fission neutron spectrum, which are still used today, are the Maxwellian and Watt spectrum representations with parameters that are adjusted to optimally reproduce the experimental spectrum for a given fissioning system at a given excitation energy. The Maxwellian spectrum is given by

$$
N(E)=\left(2 / \pi^{1 / 2} T_{M}^{3 / 2}\right) E^{1 / 2} \exp \left(-E / T_{M}\right),
$$

where the single (temperature) parameter appearing, $T_{M}$, is related to the average energy (first moment) of the spectrum $\langle E\rangle$ by

$$
<E>=(3 / 2) T_{M} .
$$

The Maxwellian spectrum is popular in applied physics because it is a closed-form expression containing a single parameter. However, this spectrum neglects the distribution of fissionfragment excitation energy, the energy dependence of the inverse process of compound nucleus formation, and the motion of the fission fragments emitting the neutrons. Therefore, $T_{M}$ is greater than the fragment temperatures physically occurring. In practice, $T_{M}$ is reduced to reproduce the tail of the experimental spectrum. To preserve normalization, this simultaneously increases $N(E)$ at lower energies in agreement with experimental measurements of the spectrum, but for the wrong physical reasons. Thus, there is no predictive power in this approach.

The two-parameter Watt spectrum ${ }^{1}$ consists of a center-of-mass Maxwellian spectrum that has been transformed ${ }^{2}$ to the laboratory system for an average fission fragment moving with an average kinetic energy per nucleon $E_{f}$. This spectrum is given by

$$
N(E)=\frac{\exp \left(-E_{f} / T_{W}\right)}{\left(\pi E_{f} T_{W}\right)^{1 / 2}} \exp \left(-E / T_{W}\right) \sinh \left[2\left(E_{f} E\right)^{1 / 2} / T_{W}\right],
$$


where $E_{f}$ and the Watt temperature $T_{W}$ are related to the average energy of the spectrum $<E>$ by

$$
<E>=E_{f}+(3 / 2) T_{W} \text {. }
$$

The Watt spectrum also neglects the distribution of fission-fragment excitation energy and the energy dependence of the inverse process of compound nucleus formation, but does account for the motion of an average fragment. However, for spontaneous fission and low-energy $\left(E_{n}<15 \mathrm{MeV}\right)$ neutron-induced fission, the concept of an average fragment is generally not a good one because there are usually two average fragment masses and two average fragment kinetic energies due to the double-humped fragment mass distribution. For these reasons, the Watt spectrum, although it is more physical than the Maxwellian spectrum, has little predictive power. Note that if one insists on using a Watt representation, the average of the separate Watt spectra corresponding to the light and heavy fragment mass yield peaks should be taken to represent the total laboratory spectrum $N(E)$. This is then a three-parameter representation assuming statistical equilibrium exists between the nascent fragments.

At the same time that these early representations were introduced for $N(E)$, the average prompt neutron multiplicity $\bar{\nu}_{p}$ was modeled ${ }^{3}$ by a simple polynomial (usually linear) in the incident neutron energy $E_{n}$, for each fissioning system considered: $\bar{\nu}_{p}=\nu_{0}+\alpha E_{n}$, and again, the parameters appearing were, and are, adjusted to optimally reproduce the experimental values.

To summarize, none of the approaches described above can be used to predict $N(E)$ and/or $\bar{\nu}_{p}$ for a different fissioning nucleus or for a different excitation energy from what has been experimentally measured. That is, there is very little predictive power in the approaches just described.

\section{Modern Approaches to the Calculation of $N(E)$ and $\bar{\nu}_{p}$}

In recent years three new theoretical approaches have evolved for the calculation of the prompt fission neutron spectrum $N(E)$. These are the following:

- The Los Alamos approach, ${ }^{4}$ begun in 1979 , which is based upon standard nuclear evaporation theory ${ }^{5}$ and simultaneously treats the average prompt neutron multiplicity $\bar{\nu}_{p}$. This approach emphasizes predictive capabilities while requiring minimal input. Refinements that treat the entire fission-fragment mass and charge distributions, instead of averages over their peak regions, have been implemented. ${ }^{6,7}$

- The Dresden approach, ${ }^{8}$ begun in 1982 , which is also based upon standard nuclear evaporation theory, ${ }^{5}$ but accounts explicitly for "neutron cascade emission." This approach emphasizes a complete description, requiring a substantial amount of experimental information. The Dresden group has also employed the Los Alamos approach including the refinements mentioned above. ${ }^{9,10}$

- The Hauser-Feshbach statistical model ${ }^{11}$ approach which accounts explicitly for the competition between neutron emission and gamma-ray emission in a given fission frag- 
ment. This approach, if properly applied, accounts for the influence of angular momentum and parity on neutron and gamma-ray emission whereas the Los Alamos and Dresden approaches do not.

\subsection{Summary of Los Alamos Model}

The original Los Alamos model ${ }^{4}$ addresses both neutron-induced and spontaneous fission and accounts for the physical effects of

(a) the distribution of fission-fragment excitation energy,

(b) the energy dependence of the inverse process of compound nucleus formation,

(c) the center-of-mass motion of the fission fragments, and

(d) multiple-chance fission at high incident neutron energy.

To simulate the initial distribution of fission-fragment excitation energy and subsequent cooling as neutrons are emitted, a triangular approximation is used to approximate the corresponding fission-fragment residual nuclear temperature distribution. This approximation, based upon the observations of Terrell, ${ }^{12}$ has a maximum temperature $T_{m}$ that is related to the initial total average fission-fragment excitation energy $<E^{*}>$ by

$$
T_{m}=\left(<E^{*}>/ a\right)^{1 / 2}
$$

where $a$ is the nuclear level-density parameter. In Eq.(5), the initial total average fissionfragment excitation energy is given by

$$
<E^{*}>=<E_{r}>+E_{n}+B_{n}-<E_{f}^{\text {tot }}>,
$$

where $\left\langle E_{r}\right\rangle$ is the average energy release in fission, $B_{n}$ and $E_{n}$ are the separation and kinetic energies of the neutron inducing fission (set to zero for spontaneous fission), and $<E_{f}^{\text {tot }}>$ is the total average fission-fragment kinetic energy. These quantities are either known or can be calculated.

The energy dependence of the inverse process is treated in the center-of-mass frame by calculating the compound nucleus formation cross section $\sigma_{c}(\epsilon)$ using an optical-model potential with explicit isospin dependence to describe neutron-rich fission fragments more correctly. It has been found that the shape of $\sigma_{c}(\epsilon)$ with $\epsilon$ affects the shape of $N(E)$.

The values of the average kinetic energy per nucleon of the average light fragment $A_{L}$ and average heavy fragment $A_{H}$ are obtained using momentum conservation and are given by

$$
E_{f}^{L, H}=\left(A_{H, L} / A_{L, H}\right)\left(<E_{f}^{t o t}>/ A\right),
$$

where $A$ is the mass number of the fissioning nucleus. 
With the inclusion of these physical effects, the prompt fission neutron spectrum in the laboratory system is given by

$$
N(E)=\frac{1}{2}\left[N\left(E, E_{f}^{L}, \sigma_{c}^{L}\right)+N\left(E, E_{f}^{H}, \sigma_{c}^{H}\right)\right],
$$

where

$$
N\left(E, E_{f}, \sigma_{c}\right)=\frac{1}{2 \sqrt{E_{f}} T_{m}^{2}} \int_{\left(\sqrt{E}-\sqrt{E_{f}}\right)^{2}}^{\left(\sqrt{E}+\sqrt{E_{f}}\right)^{2}} \sigma_{c}(\epsilon) \sqrt{\epsilon} d \epsilon \int_{0}^{T_{m}} k(T) T \exp (-\epsilon / T) d T
$$

In this equation, $\epsilon$ is the center-of-mass neutron energy and $k(T)$ is the temperaturedependent normalization integral. ${ }^{4}$ If $\sigma_{c}(\epsilon)$ is constant, Eq.(9) reduces to a closed-form expression involving the exponential integral function and the incomplete gamma function.

The average prompt neutron multiplicity $\bar{\nu}_{p}$ is obtained from energy conservation and is given by

$$
\bar{\nu}_{p}=\frac{\left\langle E^{*}\right\rangle-\left\langle E_{\gamma}^{\text {tot }}\right\rangle}{\left\langle S_{n}\right\rangle+\langle\epsilon\rangle},
$$

where $\left\langle E_{\gamma}^{\text {tot }}\right\rangle$ is the total average prompt gamma-ray energy, $\left\langle S_{n}\right\rangle$ is the average fissionfragment neutron separation energy, and $\langle\epsilon\rangle$ is the average center-of-mass energy of the emitted neutrons.

There are two important connections between $N(E)$ and $\bar{\nu}_{p}$, especially for nuclear data evaluation. The first is that the maximum temperature $T_{m}$ appearing as one of three parameters in $N(E)$, also appears in the expression for $\bar{\nu}_{p}$ as $T_{m}^{2}$ through Eq. (5). The second is that the average center-of-mass neutron energy $\langle\epsilon\rangle$ appearing in the expression for $\bar{\nu}_{p}$, is also the first moment of the center-of-mass neutron spectrum $\Phi(\epsilon)$ corresponding to the laboratory neutron spectrum $N(E)$. These two connections are very significant because they mean that if one has experimental information on either $N(E)$ or $\bar{\nu}_{p}$, then that information can be used as a constraint in the calculation of the other, unmeasured, observable. We note here, that there are many more measurements of $\bar{\nu}_{p}$ than there are of $N(E)$.

The Los Alamos model has been used to calculate $N(E)$ and $\bar{\nu}_{p}$ for neutron-induced and spontaneous fission, and for unmeasured as well as measured systems.

\subsection{Summary of Dresden Model}

The Dresden model, ${ }^{8}$ currently known as the Complex Cascade Evaporation Model (CCEM), accounts for the physical effects of

(a) the distribution of fission-fragment excitation energy in each step of the cascade,

(b) the energy dependence of the inverse process of compound nucleus formation,

(c) the center-of-mass motion of the fission fragments, 
(d) the anisotropy of the center-of-mass neutron spectrum,

(e) the complete fission-fragment mass and kinetic energy distributions, and

(f) semi-empirical fission-fragment nuclear level densities.

With information on the above physical effects given in sufficient detail, the prompt fission neutron spectrum in the laboratory system is given by

$$
N(E)=\sum_{A} \int P(A, T K E) N(E, A, T K E) d T K E,
$$

where $P(A, T K E)$ is the normalized fission-fragment mass distribution for a fixed value of the total fission-fragment kinetic energy $T K E$, and $N(E, A, T K E)$ is the laboratory spectrum for fixed fragment mass $A$ and fixed $T K E$. The sum and integral are over all contributing fragment mass numbers and total kinetic energies, respectively. The fragment spectrum $N(E, A, T K E)$ is given by

$$
N(E, A, T K E)=\int_{\left(\sqrt{E}-\sqrt{E_{f}}\right)^{2}}^{\left(\sqrt{E}+\sqrt{E_{f}}\right)^{2}} \frac{\phi(\epsilon, A, T K E)}{4 \sqrt{\epsilon E_{f}}}\left\{\frac{1+b\left[\left(E-E_{f}-\epsilon\right)^{2} / 4 \epsilon E_{f}\right]}{[1+(b / 3)]}\right\} d \epsilon,
$$

where $E_{f}$ is the kinetic energy per-nucleon of the fragment, $b$ is the anisotropy coefficient, $\epsilon$ is the center-of-mass neutron energy, and $\phi(\epsilon, A, T K E)$ is the center-of-mass neutron spectrum for fixed mass and fixed $T K E$, given by

$$
\phi(\epsilon, A, T K E)=\sum_{i} \int_{B_{i}}^{\infty} \phi_{i}\left(\epsilon, E^{*}, A-i\right) P_{i}\left(E^{*}, A, T K E\right) d E^{*} .
$$

In this equation, the sum is over the steps $i$ of the cascade while the integral is over the fragment excitation energy $E^{*}$, and $B_{i}$ is the neutron binding energy in a fragment that has emitted $i$ neutrons. Then, $P_{i}\left(E^{*}, A, T K E\right)$ is the excitation energy distribution before step $i$ and is expressed in terms of $P_{i-1}$ and, ultimately, $P_{0}$, which is assumed Gaussian. Finally, $\phi_{i}$ is the center-of-mass neutron energy spectrum given by Weisskopf. ${ }^{5}$ The CCEM has been used extensively in studies of the ${ }^{252} \mathrm{Cf}(\mathrm{sf})$ reaction.

\subsection{Summary of Hauser-Feshbach Approach}

This approach consists of Hauser-Feshbach statistical model calculations of the de-excitation of representative nuclei of the fission-fragment mass and charge distributions, which are then weighted by the (known) yield distribution to construct the total neutron spectrum. This model accounts for the physical effects contained in the Los Alamos and Dresden models and, in addition, accounts for

(a) neutron and gamma-ray competition in the de-excitation of a given fission fragment,

(b) neutron transmission coefficients $T_{l j}$ from an optical-model potential for each fragment considered,

(c) gamma-ray transmission coefficients $T_{\gamma}$ for each fragment considered, and 
(d) the angular momentum distribution $P(J)$ for each fragment considered.

Due to space limitations, a detailed description of the Hauser-Feshbach formalism for deexcitation of fission fragments is not presented here. Note, however, that crucial ingredients of these calculations include isospin-dependent neutron optical potentials for fission fragments, fragment initial excitation energy and angular momentum distributions, fragment nuclear level densities, and the partition of available excitation energy between light and heavy fragments. To date, just as in the CCEM, the Hauser-Feshbach approach has been applied primarily to studies of the ${ }^{252} \mathrm{Cf}(\mathrm{sf})$ reaction.

\section{Representative Calculations from the Modern Approaches}

Calculations using the Los Alamos model are shown in Figs. 1-5. The dependence of $N(E)$ upon the fissioning nucleus and its excitation energy is shown in Figs. 1 and 2 for Eqs. (8) and (9) evaluated in the constant cross section approximation. Figure 1 shows how the spectrum increases at high energies and decreases at low energies as the mass and charge of the fissioning nucleus increases, for thermal-neutron-induced fission. This example shows that $\left\langle E_{r}\right\rangle$ is increasing faster with mass number $A$ than $\left\langle E_{f}^{\text {tot }}\right\rangle$ is increasing with charge number $Z$, of the fissioning nucleus. Similarly, Fig. 2 shows how the spectrum increases at high energies and decreases at low energies as the kinetic energy of the incident neutron increases, for the first-chance fission of ${ }^{235} \mathrm{U}$. Figure 3 compares the exact $\left[\sigma_{c}(\varepsilon)\right.$ calculated from an optical potential] and approximate $\left[\sigma_{c}=\right.$ constant] versions of the Los Alamos spectrum with experimental data. Clearly, there is a preference for the exact energy-dependent cross section calculation. The Los Alamos model has also been used to calculate the neutroninduced multiple-chance fission neutron spectrum for ${ }^{235} \mathrm{U}$ up through third-chance fission. ${ }^{4,6}$ The resulting fission neutron spectrum matrix $N\left(E, E_{n}\right)$, divided by the thermal spectrum $N(E, 0)$, is shown in Fig. 4. Here, the partition of the total available excitation energy into neutron emission prior to fission and neutron emission from accelerated fission fragments leads to suggestions of a staircase effect in the peak regions of the matrix and an oscillatory effect in the tail regions of the matrix. The former effect is due largely to the pre-fission evaporation neutrons while the latter effect is due largely to the occurence of cooler fission fragments following the emission of a neutron, or two neutrons, prior to fission. Figure 5 shows the application of the Los Alamos model to the calculation of the energy and angle spectrum $N(E, \Theta)$ for the ${ }^{252} \mathrm{Cf}(\mathrm{sf})$ reaction, by the Dresden group. ${ }^{13}$ The calculated spectrum, from a refined Los Alamos model (their GMNM model ${ }^{9}$ ), is shown in the upper portion of the figure while the smoothed experimental data ${ }^{14}$ is shown in the lower portion of the figure. The good overall agreement is a rather remarkable achievement by the Dresden group.

Two calculations using the Dresden CCEM are shown in Fig. 6 for the ${ }^{252} \mathrm{Cf}(\mathrm{sf})$ reaction. In this figure the calculations and experimental data are shown as deviations from a Maxwellian spectrum. The Dresden model(CEM), solid curves from Eqs. (11)-(13), for a center-of-mass anisotropy coefficient $b=0.1(\beta=0.1)$ agrees with experiment much better than the corresponding curve with $b=0.0(\beta=0.0)$, especially at the low energy end of the spectrum. Clearly, the anisotropy of the center-of-mass spectrum must be taken into account to obtain 
the most realistic description of the experimental spectrum. ${ }^{15}$

Two calculations using the Hauser-Feshbach approach for the ${ }^{252} \mathrm{Cf}(\mathrm{sf})$ reaction are shown in Figs. 7 and 8 . Gerasimenko and Rubchenya ${ }^{16}$ consider 18 representative fission fragments, and use a Fermi-gas level density and a Gaussian distribution of initial excitation energy, to obtain the total spectrum shown in good agreement with experiment in Fig. 7. They obtain even better agreement when including a center-of-mass anisotropy coefficient of $b=0.15$, although this effect is still under study. Seeliger et al. ${ }^{17}$ have shown in their Hauser-Feshbach calculations that for the right value of a "scaling factor" on the gamma-emission width, the laboratory neutron energy spectrum and neutron total angular distribution are well reproduced. This is shown in Fig. 8 for the neutron total angular distribution.

The many other calculational examples of the modern approaches that exist in the literature are not shown here due to space limitations.

\section{Conclusions}

It is concluded that prompt fission neutron spectra and average prompt neutron multiplicities can be calculated with reasonably good confidence for unmeasured as well as measured systems, and for neutron-induced as well as spontaneous fission. Current limitations to calculating $N(E), \bar{\nu}_{p}, N\left(E, E_{n}\right)$, and $\bar{\nu}_{p}\left(E_{n}\right)$ with higher accuracy than is now possible include insufficiently accurate information on

- excitation energy partition in fission,

- fission fragment nuclear level densities,

- isospin dependence of global neutron optical-model potentials,

- fission fragment ground-state masses (for calculating $<E_{r}>$ ),

- fission fragment mass and charge distributions, and

- fission fragment initial excitation energy and initial angular momentum distributions.

It is believed that, ultimately, the Hauser-Feshbach approach will probably yield the most accurate results in the calculation of the prompt fission neutron spectrum and the average prompt neutron multiplicity. This is because the simultaneous calculation of neutron and gamma-ray emission in competition is the best way to account for the dissipation of the total fission-fragment excitation energy. A second reason is the detail inherent in the HauserFeshbach approach: explicit treatment of each fragment in a relatively large number of fissionfragment pairs. Thus, a Hauser-Feshbach approach will presumably lead to simultaneous physically accurate calculations of $N\left(E, E_{n}\right)$ and $\bar{\nu}_{p}\left(E_{n}\right)$ for neutron-induced fission, and $N(E)$ and $\bar{\nu}_{p}$ for spontaneous fission, together with $N_{\gamma}\left(E, E_{n}\right)$ and $\bar{\nu}_{\gamma p}\left(E_{n}\right)$ for neutroninduced fission, and $N_{\gamma}(E)$ and $\bar{\nu}_{\gamma p}$ for spontaneous fission, respectively.

\section{References}


1. B. E. Watt, Phys.Rev. 87, 1037 (1952).

2. N. Feather, "Emission of Neutrons from Moving Fission Fragments," BM-148, British Mission (1942).

3. F. Manero and V. A. Konshin, Atomic Energy Rev. 10, 637 (1972).

4. D. G. Madland and J. R. Nix, Nucl. Sci. Eng. 81, 213 (1982).

5. V. F. Weisskopf, Phys. Rev. 52, 295 (1937).

6. D. G. Madland, R. J. LaBauve, and J. R. Nix, Proceedings of the IAEA Consultants' Meeting on the Physics of Neutron Emission in Fission, Mito, Japan, 1988, H. D. Lemmel, Ed. [IAEA, INDC(NDS)-220, 1989], p. 259.

7. D. G. Madland, Proceedings of the International Conference on Nuclear Data for Science and Technology, Mito, Japan, 1988, S. Igarasi, Ed. (Saikon Publishing, Tokyo, 1988), p. 759.

8. H. Märten and D. Seeliger, J. Phys. G 10, 349 (1984).

9. H. Märten and D. Seeliger, Nucl. Sci. Eng. 93, 370 (1986).

10. H. Märten, A. Ruben, and D. Seeliger, Proceedings of the IAEA Consultants' Meeting on the Physics of Neutron Emission in Fission, Mito, Japan, 1988, H. D. Lemmel, Ed. [IAEA, INDC(NDS)-220, 1989], p. 245.

11. W. Hauser and H. Feshbach, Phys. Rev. 87, 366 (1952).

12. J. Terrell, Phys. Rev. 113, 527 (1959).

13. D. Seeliger, H. Märten, and A. Ruben, Contributions to the Theory of Fission Neutron Emission [INDC(GDR)-057, Vienna, 1990].

14. H. Märten et al., Nucl. Instr. Meth. A264, 375 (1988).

15. H. Märten and D. Seeliger, Proceedings of the Advisory Group Meeting on Nuclear Standard Reference Data, Geel, Belgium, 1984, (IAEA-TECDOC-335, Vienna, 1985), p. 255.

16. B. F. Gerasimenko and V. A. Rubchenya, Proceedings of the Advisory Group Meeting on Properties of Neutron Sources, Leningrad, USSR, 1986, (IAEA-TECDOC-410, Vienna, 1987), p. 208.

17. D. Seeliger, et al., Prompt Neutron Emission in Nuclear Fission, [INDC(GDR)-056/L, Vienna, 1989], p. 8. 